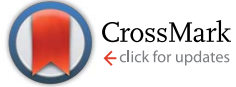

Cite this: Chem. Sci., 2016, 7, 3556

Received 19th December 2015 Accepted 12th February 2016

DOI: $10.1039 / \mathrm{c} 5 \mathrm{sc} 04902 d$

www.rsc.org/chemicalscience

\section{Insight into the mechanism and outcoupling enhancement of excimer-associated white light generation $\uparrow$}

\author{
Ying-Hsiao Chen, $\dot{t}^{a}$ Kuo-Chun Tang, $t_{t^{a}}$ Yi-Ting Chen, ${ }^{a}$ Jiun-Yi Shen, ${ }^{a} \mathrm{Yu}-\mathrm{Sin} \mathrm{Wu},{ }^{a}$ \\ Shih-Hung Liu, ${ }^{a}$ Chun-Shu Lee, ${ }^{\mathrm{b}}$ Chang-Hsuan Chen, ${ }^{\mathrm{b}}$ Tzu-Yu Lai, ${ }^{c}$ \\ Shih-Huang Tung, ${ }^{c}$ Ru-Jong Jeng, ${ }^{* c}$ Wen-Yi Hung, ${ }^{* b}$ Min Jiao, ${ }^{d}$ Chung-Chih Wu ${ }^{d}$ \\ and Pi-Tai Chou ${ }^{* a}$
}

Fundamental insight into excimer formation has been gained by using 9,10-bis[4-(9-carbazolyl)phenyl] anthracene] $\left(\mathrm{Cz}^{9} \mathrm{PhAn}\right)$ as a probe. $\mathrm{Cz}^{9} \mathrm{PhAn}$ exhibits a highly emissive blue fluorescence in solution and is found to emit a panchromatic white light spectrum $(400-750 \mathrm{~nm})$ in film, powder and single crystal, in which an additional excimer band appears at $\sim 550 \mathrm{~nm}$. Detailed structural analyses, emission relaxation dynamics and a theoretical approach conclude the formation of an anthracene*/phenyl ring excimer through an overlap between $\pi^{*}$ (anthracene) and $\pi$ (phenyl ring) orbitals in a face-to-edge stacking orientation. The rate of excimer formation is determined to be $2.2 \times 10^{9} \mathrm{~s}^{-1}$ at room temperature, which requires coupling with lattice motion with an activation energy of $0.44 \mathrm{kcal} \mathrm{mol}^{-1}$. Exploiting $\mathrm{Cz}^{9} \mathrm{PhAn}$ as a single emitter, a fluorescent white organic light emitting diode (WOLED) is fabricated with a maximum external quantum efficiency $\left(\eta_{\text {ext }}\right)$ of $3.6 \%$ at $1000 \mathrm{~cd} \mathrm{~m}^{-2}(4.2 \mathrm{~V})$ and Commission Internationale de L'Eclairage (CIE) coordinates of $(0.30,0.33)$. The white-light $\mathrm{Cz}^{9} \mathrm{PhAn}$ reveals a preferred orientation of the transition dipole moment in the emitting layer to enhance light outcoupling. This non-doped, single component ( $\left.\mathrm{Cz}^{9} \mathrm{PhAn}\right)$ WOLED greatly reduces the complexity of the fabrication process, rendering a green and cost-effective alternative among the contemporary display/lighting technologies.

\section{Introduction}

White organic light-emitting diodes (WOLEDs) have attracted broad attention for potential applications in flat-panel displays and solid-state lighting. ${ }^{1}$ WOLEDs can be engineered with single or multiple active layers to attain white light emission. Multiple active layer designs normally incorporate different chromophores into each active layer, while multiple chromophores are commonly required and mixed in the single active layer design. Sun et al. reported a WOLED device using a multiple active-layer structure with a blue fluorescent dopant

\footnotetext{
${ }^{a}$ Department of Chemistry, National Taiwan University, Taipei, 10617 Taiwan, Republic of China. E-mail: chop@ntu.edu.tw

${ }^{b}$ Institute of Optoelectronic Sciences, National Taiwan Ocean University, Keelung 20224, Taiwan, Republic of China.E-mail:wenhung@mail.ntou.edu.tw

'Institute of Polymer Science and Engineering, National Taiwan University, Taipei, 10617 Taiwan, Republic of China.E-mail: rujong@ntu.edu.tw

${ }^{d}$ Department of Electrical Engineering, Graduate Institute of Electronics Engineering, and Graduate Institute of Photonics and Optoelectronics, National Taiwan University, Taipei 10617, Taiwan, Republic of China

$\dagger$ Electronic supplementary information (ESI) available. CCDC 1008747. For ESI and crystallographic data in CIF or other electronic format see DOI: 10.1039/c5sc04902d

\$ Y.-H. Chen and K.-C. Tang contributed equally to this work.
}

and phosphorescent dopants (for green and red). This device exhibited high internal efficiency ( 100\%) with a color rendering index (CRI) of 85 and Commission Internationale de L'Eclairage (CIE) coordinates of $(0.40,0.41){ }^{2}$ More recently, Zhou et al. have reported a device based on the blue fluorescence of $4,4^{\prime}$-bis-[ $N$-(1-naphthyl)- $N$-phenylamino]biphenyl (NPB), with the green and red phosphorescence of a platinum complex to duplicate sunlight, exhibiting an extraordinarily high CRI of 97.3 Wong and co-workers reported a bipolar universal host, oOXA, combining RGB phosphors within a single emitting layer to realize WOLEDs with external quantum efficiency of $20 \%$, high CRI of 87 and stable chromaticity. ${ }^{4}$

In addition to the mixing of RGB colors, white light can in fact be simply generated by ratiometric fine-tuning of two complementary colors of light, for example, blue and yellow, on the CIE color space. Park and co-workers reported a WOLED comprising the covalently linked blue and yellow color-emitting fluorophores of proton transfer molecules., ${ }^{5,6}$ Chou et al. reported a pure white-light emitting mechanism developed by anchoring organic yellow-light-emitting fluorophores onto inorganic ultra-small CdSe quantum dots (blue-light emitting). ${ }^{7}$ However, both approaches, the use of RGB and two complementary colors, require a delicate balance for both excitation 
and emission between mixed multiple materials. In particular, color balancing becomes much more complicated when the inter-chromophore energy transfer mechanism is involved. These, together with the various aging lifespans of each emissive material, which may cause spectral variation collaterally, would certainly hamper any practical application.

Thus, the development of a single emitting material capable of emitting white light, preferably panchromatic white light, is demanding. As for harvesting phosphorescent white light, a single-doped WOLED with $20 \%$ external efficiency has been achieved $;^{8 b}$ it is based on a phosphorescent Pt complex in a relatively complicated device structure: a co-host of TAPC : PO15 (1:1) for a 10\%-doped Pt device. White light was generated by coupling blue-green phosphorescence from single $\mathrm{Pt}$ complexes and orange emission from $\mathrm{Pt}$ aggregates. However, phosphorescent OLEDs (PHOLEDs) are hampered by the high cost and rarity of the noble metal elements. Alternatively, WOLEDs employing metal-free single-emitters have emerged as cheaper substitutes for PHOLEDs and there has been rapid development of these into single-emitter fluorescent WOLEDs by various methods such as $\mathrm{pH}$-dependent emission, ${ }^{9 a}$ thermo-responsive emission ${ }^{9 b}$ and intermolecular charge transfer (ICT). ${ }^{9 c}$

In light of pursuing single-molecule-active-layered fluorescent WOLEDs, we recently reported a single-molecule white light-emitting system, 1-hydroxy-11H-benzo[b]fluoren-11-one, ${ }^{10}$ based on its reversible excited-state intramolecular proton transfer reaction, such that both normal (blue) and protontransfer tautomer (yellow) emissions appear to give white light with CIE coordinates at $(0.30,0.27)$. Among other WOLEDs fabricated from various single-component materials, ${ }^{11-16}$ carbazole derivative-based devices have been demonstrated to have great color purity. ${ }^{11-13}$ For instance, Hou and coworkers developed two carbazole-substituted aromatic enynes as singlewhite light emitting materials in a double-layer device structure. ${ }^{12}$ Moreover, anthracene-cored compounds were utilized not only for blue emitting derivatives but also for white emitting frameworks due to their excellent high photoluminescence (PL) quantum efficiency and good thermal stability. ${ }^{14-19}$ Zhang and coworkers synthesized a triphenylamine/pyrenylanthracenecomposed material, DPAA, which was fabricated as the emitting layer in a three-layer device exhibiting white light emission. The visible light emission spectra of these materials were commonly derived from normal (monomer) and excimer fluorescence bands. Our recent advance revealed that the color purity and the efficiency of the OLED device can be fine-tuned by modifying the position of the carbazole moiety on the anthracene derivative and/or the $\pi$-spacer conjugation length between the carbazole moiety and anthracene. ${ }^{20,21}$ Although these carbazolesubstituted anthracene derivatives exhibited only blue emission suitable for non-doped blue devices, it is feasible, on the basis of these in-depth findings, to tailor and fine-tune the molecular structure in an aim to attain panchromatic light with high quantum efficiencies.

We report herein the synthesis (see ESI $\dagger$ ), characterization, and WOLED application of a new material, $\mathbf{C z}^{\mathbf{9}} \mathbf{P h A n}$ (Scheme 1), comprising two 9-phenylcarbazole units covalently linked to

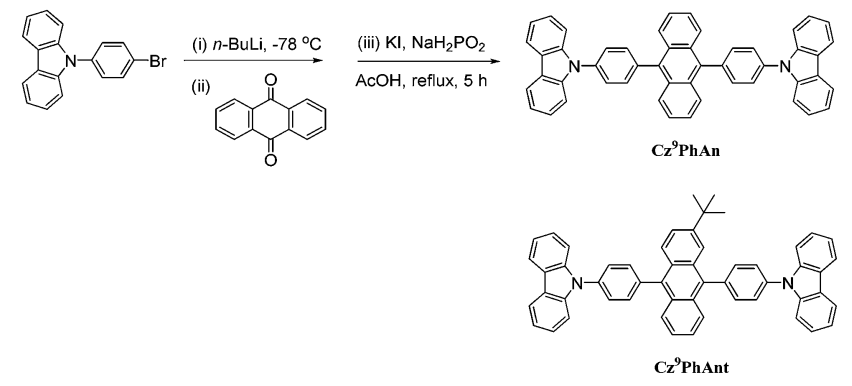

Scheme 1 Synthetic routes toward $\mathrm{Cz}^{9} \mathrm{PhAn}$. Also shown is the structure of a $\mathrm{Cz}^{9} \mathrm{PhAn}$ analogue, $\mathrm{Cz}{ }^{9} \mathrm{PhAnt}$, for comparison.

the $\mathrm{C} 9$ and $\mathrm{C} 10$ positions of anthracene. $\mathbf{C z}^{\mathbf{9}} \mathbf{P h A n}$ exhibits prominent excimer emission in single crystal and solid film. While excimer generation may not be uncommon in lighting devices, the fundamental insight into the mechanism of excimer-associated white light generation is still pending. The goal of this study is thus to gain understanding of the fundamentals of excimer formation via structural analyses and the associated spectroscopy and reaction dynamics. Furthermore, the combination of dual, far-separated and high-intensity monomer (450 $\mathrm{nm})$ and excimer $(540 \mathrm{~nm})$ emissions in $\mathbf{C z}^{\mathbf{9}} \mathbf{P h A n}$ permits remarkable white light generation. As a result, a non-doped WOLED incorporating $\mathbf{C z}^{\mathbf{9}} \mathbf{P h A n}$ as the emitter is successfully fabricated, showing a high value of $\eta_{\text {ext }}$ of $3.6 \%$ at $1000 \mathrm{~cd} \mathrm{~m}^{-2}$ with CIE coordinates of $(0.30,0.33)$, which should be more attractive than doped WOLEDs because the fabrication processes are facile and the devices are more reliable. Details of results and discussion are elaborated as follows.

\section{Results and discussion}

Fig. 1a shows the steady-state absorption and emission spectra of $\mathbf{C z}^{\mathbf{9}} \mathbf{P h A n}$ dissolved in dichloromethane. Pertinent photophysical properties are summarized in Table 1 . The lowest lying absorption band is at $\lambda_{\max } \sim 396 \mathrm{~nm}$ with vibronic structures, and the emission band peaks at $430 \mathrm{~nm}$. The absorption and emission spectra show a good mirror-image relationship with a common Stokes shift (difference in frequency between lowestlying absorption peak and emission peak) of $\sim 2000 \mathrm{~cm}^{-1}$. The measured emission (fluorescence) quantum yield of $\mathbf{C z}^{9} \mathbf{P h A n}$ in dichloromethane is near unity ( 1.0). Further time-resolved fluorescence measurements (see Fig. 2a) revealed a single exponential fluorescence decay with a time constant of $4.1 \mathrm{~ns}$ $\left(\chi^{2}=1.02\right)$. Accordingly, the radiative decay rate constant was then calculated to be as large as $2.4 \times 10^{8} \mathrm{~s}^{-1}$, implying that the lowest lying absorption is a fully allowed $\pi-\pi^{*}$ transition. The absorption and emission spectra of a $\mathbf{C z}^{\mathbf{9}} \mathbf{P h A n}$ film sample (prepared by thermal evaporation) are also shown in Fig. 1a. Both the absorption (peak at $404 \mathrm{~nm}$ ) and normal emission (peak at $445 \mathrm{~nm}$ ) spectra are slightly red-shifted with respect to those in dichloromethane solution. Remarkably, however, an additional emission band appears with a peak wavelength at $550 \mathrm{~nm}$. The excitation scan, monitored at either $445 \mathrm{~nm}$ or 550 $\mathrm{nm}$, shows an identical excitation spectral profile, which also 


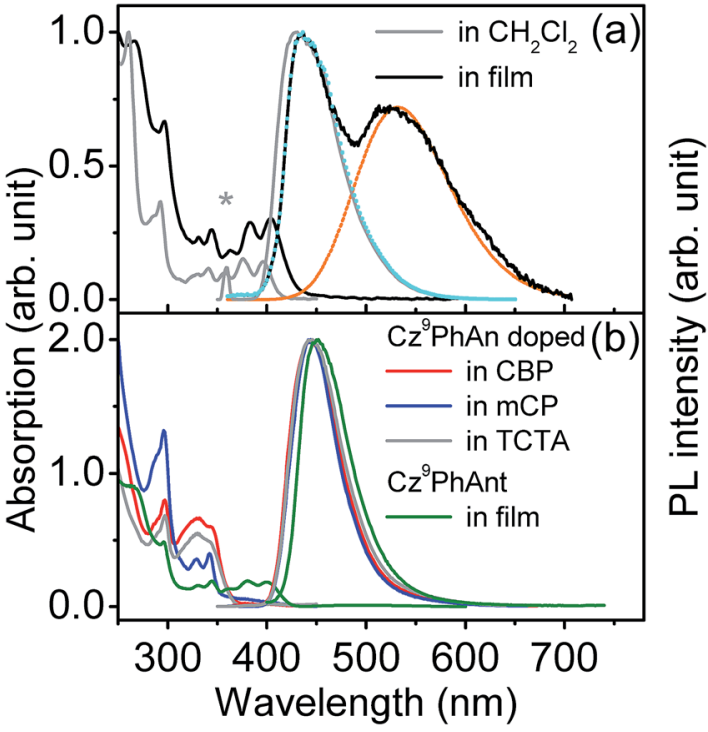

Fig. 1 (a) Steady-state absorption and photoluminescence spectra of $\mathrm{Cz}^{9} \mathrm{PhAn}$ in $\mathrm{CH}_{2} \mathrm{Cl}_{2}$ and film (prepared via thermal evaporation). Blue and orange dashed lines represent the spectral decomposition of the film photoluminescence spectrum (see text for assignments and details). The asterisk shows the excitation wavelength. (b) Steady-state spectra of $\mathrm{Cz}^{9} \mathrm{PhAnt}$ film and $3 \mathrm{wt} \% \mathrm{Cz}^{9} \mathrm{PhAn}$ doped in a common host (prepared via thermal evaporation).

resembles the absorption spectrum, indicating that both the $445 \mathrm{~nm}$ and $550 \mathrm{~nm}$ emission bands originate from a common ground state. Accordingly, the assignment of this $550 \mathrm{~nm}$ band in the $\mathbf{C z}^{\mathbf{9}} \mathbf{P h A n}$ solid film to an excimer emission is reasonable.

Further support of this viewpoint is given by the dilution of the $\mathbf{C z}^{\mathbf{9}} \mathbf{P h A n}$ solid film. Upon doping of $\mathbf{C z}^{\mathbf{9}} \mathbf{P h A n}$ onto a host material such as 4,4'-bis( $N$-carbazolyl)-1,1'-biphenyl (CBP), 1,3bis $\left(N\right.$-carbazolyl)benzene $(\mathrm{mCP})$ and $4,4^{\prime}, 4^{\prime \prime}$-tri $(N$-carbazolyl)triphenylamine (TCTA), forming a diluted $\mathbf{C z}^{\mathbf{9}} \mathbf{P h A n}$ solid film (3\% by weight), only $445 \mathrm{~nm}$ emission appears, and no $550 \mathrm{~nm}$ emission band (see Fig. 1b and Table 1). Perhaps the firmest support of excimer formation is given by the time-resolved emission analyses of a $\mathbf{C z}^{\mathbf{9}} \mathbf{P h A n}$ film sample shown in Fig. $2 \mathrm{~b}$ and c. Time-correlated single photon counting (TCSPC) measurements monitored at $440 \mathrm{~nm}$ (Fig. 2b) show a short emission decay time of $0.45 \mathrm{~ns}(450 \pm 30 \mathrm{ps})$, which is in sharp contrast ( $\sim 10$ times faster) to the fluorescence decay of $\mathbf{C z}^{\mathbf{9}} \mathbf{P h A n}$ in $\mathrm{CH}_{2} \mathrm{Cl}_{2}$ (Fig. 2a) as well as $\mathbf{C z}^{\mathbf{9}} \mathbf{P h A n}$ doped (3\% by weight) in
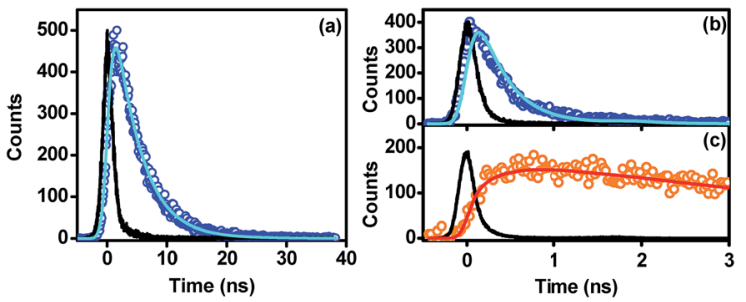

Fig. 2 Time-resolved photoluminescence decay curves of $\mathrm{Cz}^{9} \mathrm{PhAn}$ in dichloromethane solution monitored at $430 \mathrm{~nm}$ (a), and $\mathrm{Cz}^{9} \mathrm{PhAn}$ film sample monitored at $440 \mathrm{~nm}$ (b) and $600 \mathrm{~nm}$ (c). Open circles denote TCSPC data points, while solid lines denote the poly-exponential fitting of the data, except for the black lines, which represent instrument response functions. The excitation wavelength is $380 \mathrm{~nm}$. Fitting results are summarized in Table 1.

CBP, mCP and TCTA films (see Table 1 and Fig. S1 in the ESI $\dagger$ ). On the other hand, as shown in Fig. 2c, the time-resolved emission decay monitored at $600 \mathrm{~nm}$ clearly reveals a rise and a decay component, whose time constants were fitted to be 470 $\pm 30 \mathrm{ps}$ and $4.4 \mathrm{~ns}$, respectively. Notably, within experimental error, the rise time of the excimer emission band matches well with the decay time of the normal emission band. The associated kinetics can thus be well described by a precursorsuccessor type of relationship between monomer (precursor) and excimer (successor) emission. With the confirmation of the excimer formation and subtraction of the normal (monomer) emission profile obtained from the diluted film spectra (Fig. 1b), we were able to spectrally decompose the film spectrum into normal (monomer) and excimer photoluminescence and hence determine their corresponding emission quantum yields (see blue and orange curves in Fig. 1a).

Fig. 3 schematically summarizes the excited state dynamics and the emission of both monomeric and excimeric excitons in the solid state upon photoexcitation. In diluted $\mathrm{CH}_{2} \mathrm{Cl}_{2}$ solution and $\mathbf{C z}^{\mathbf{9}} \mathbf{P h A n}\left(3 \mathrm{wt} \%\right.$ ) doped solid film, the lifetime of $\mathbf{C z}^{\mathbf{9}} \mathbf{P h A n}$ in the excited state is much shorter than its diffusion time, explaining the absence of excimer emission. As for the solid $\mathbf{C z}^{9} \mathbf{P h A n}$, excimer formation via exciton-diffusion is feasible for the molecules that are in close contact with each other due to tight molecular packing. Temperature-dependent photoluminescence and the associated relaxation dynamics were then studied to gain the detailed kinetic parameters of the excimer formation. Upon lowering the temperature from $298-77 \mathrm{~K}$ in

Table 1 Photophysical Properties of $\mathrm{Cz}^{9} \mathrm{PhAn}$

\begin{tabular}{|c|c|c|c|c|c|}
\hline $\mathrm{Cz}^{9} \mathbf{P h A n}$ & Solution $\left(\mathrm{CH}_{2} \mathrm{Cl}_{2}\right)$ & Neat film & $\begin{array}{l}\text { Doped in film } \\
\text { of CBP }\end{array}$ & $\begin{array}{l}\text { Doped in film } \\
\text { of } \mathrm{mCP}\end{array}$ & $\begin{array}{l}\text { Doped in film } \\
\text { of TCTA }\end{array}$ \\
\hline$\lambda_{\mathrm{Abs}}(\mathrm{nm})$ & 396 & 404 & 404 & 404 & 404 \\
\hline$\phi$ Q.Y. & $\sim 1$ & $0.25 ; 0.22$ & $\sim 1$ & $\sim 1$ & $\sim 1$ \\
\hline$\tau_{\mathrm{Em}}{ }^{a}(\mathrm{~ns})$ & 4.1 & $0.45 ; 4.4^{c}$ & 3.9 & 4.5 & 4.2 \\
\hline$k_{\mathrm{r}}^{b}\left(\mathrm{~s}^{-1}\right)$ & $2.4 \times 10^{8}$ & $5.6 \times 10^{8} ; 6.7 \times 10^{7 d}$ & $2.6 \times 10^{8}$ & $2.2 \times 10^{8}$ & $2.4 \times 10^{8}$ \\
\hline
\end{tabular}

${ }^{a} \tau_{\text {Em: }}$ fitted emission lifetime. ${ }^{b} k_{\mathrm{r}}$ : calculated radiative decay rate constant. ${ }^{c}$ Accompanied with an initial fast rise component whose time constant is fitted to $0.47 \mathrm{~ns}(470 \mathrm{ps}){ }^{d}$ A lower limit calculated with the assumption of the upper limit of branching (0.75) of excimer formation. 

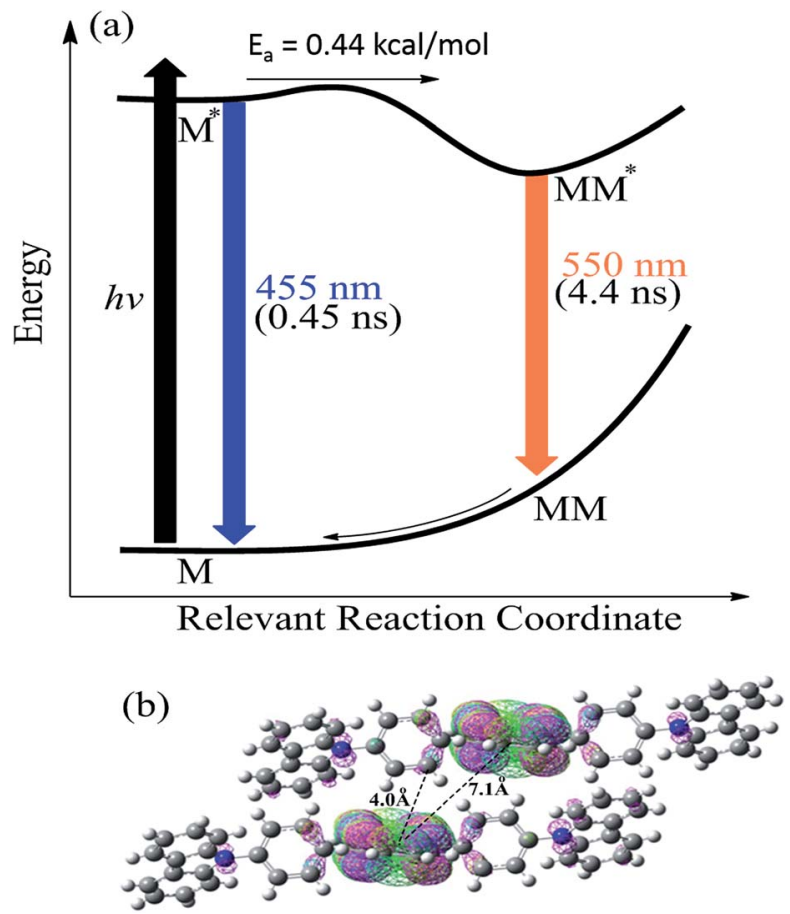

Fig. 3 (a) A schematic diagram illustrating the photodynamics of $\mathrm{Cz}^{9} \mathrm{PhAn}$ solid thin film upon photoexcitation. $E_{\mathrm{a}} 0.44\left(\mathrm{kcal} \mathrm{mol}^{-1}\right)$ : the activation energy of excimer formation. (b) The side view of two $\mathrm{Cz}^{9} \mathrm{PhAn}$ slabs for which excimer formation may be associated with $M-M *$ ( $M$ denotes $C z^{9} \mathrm{PhAn}$ ) interaction assisted by the lattice motion. Also shown are the face-to-edge frontier orbitals of two $\mathrm{Cz}^{9} \mathrm{PhAn}$ slabs. HOMO is in pink and LUMO is in green.

neat $\mathbf{C z}^{\mathbf{9}} \mathbf{P h A n}$ film, as shown in (Fig. 4), the results clearly show that the excimer $550 \mathrm{~nm}$ emission band gradually decreases, accompanied by an increase in the monomer $440 \mathrm{~nm}$ emission. Correspondingly, the decay rate constant $k_{\text {neat }}(T)$ of the
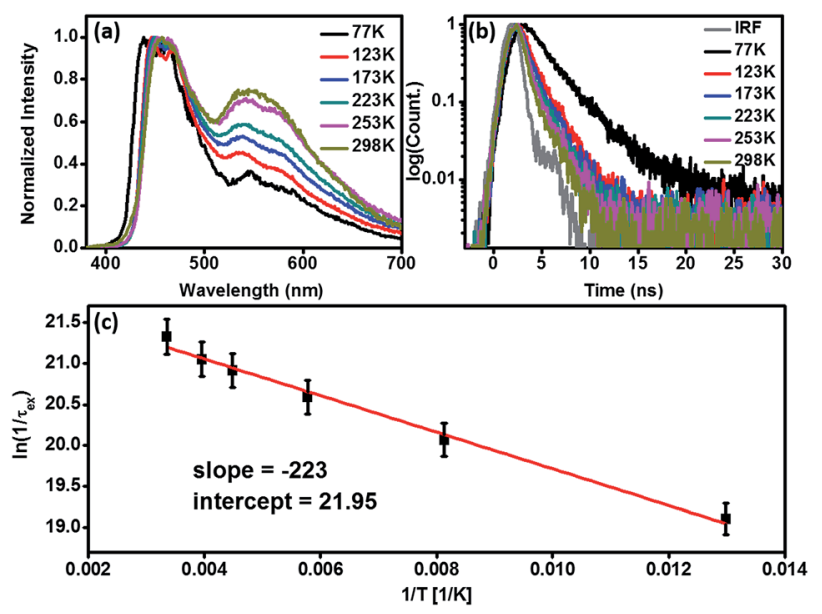

Fig. 4 Temperature-dependent properties of neat $\mathrm{Cz}^{9} \mathrm{PhAn}$ film (prepared via thermal evaporation). (a) Photoluminescence spectra, note: the emission is normalized at the maximum of the monomer emission (445 nm) for comparison. (b) Time-resolved photoluminescence decay curves monitored at $440 \mathrm{~nm}$. (c) Arrhenius plot of the rate constant $\left(\mathrm{l} / \tau_{\mathrm{ex}}\right)$ versus reciprocal temperature. monomer emission increases from 0.45 (298 K) to $2.2 \mathrm{~ns}(77 \mathrm{~K})$, supporting the thermally activated excimer formation. We also measured the temperature-dependent relaxation dynamics of the $3 \mathrm{wt} \% \mathbf{C z}^{\mathbf{9}} \mathbf{P h A n}$ doped film, i.e., $k_{\text {dilute }}(T)$, where only normal emission is resolved. The temperature-dependent rate constant of the excimer formation $k_{\text {ex }}(T)$ can thus be deduced using eqn (1) and an Arrhenius plot for $\ln k_{\text {ex }}(T)$ as a function of $1 / T$ can be expressed in eqn (2), where $E_{\mathrm{a}}$ and $A$ denote the reaction activation energy and frequency factor, respectively.

$$
\begin{gathered}
k_{\text {ex }}(T)=k_{\text {neat }}(T)-k_{\text {dilute }}(T) \\
\ln k_{\text {ex }}(T)=\ln A-\frac{E_{\mathrm{a}}}{R T}
\end{gathered}
$$

The results shown in Fig. 4c reveal a straight line and consequently, $E_{\mathrm{a}}$ and $A$ for the excimer formation process are calculated to be $0.44 \mathrm{kcal} \mathrm{mol}^{-1}$ and $3.41 \times 10^{9} \mathrm{~s}^{-1}$, respectively. The small frequency factor $\left(\sim 30 \mathrm{~cm}^{-1}\right)$ indicates that the low frequency motion, most likely the motion associated with lattice vibration, would induce the excimer formation.

As shown in Fig. S2 of the ESI, $\dagger \mathbf{C z}^{\mathbf{9}} \mathbf{P h A n}$ in a single crystal also exhibits dual emission maximized at 450 and $560 \mathrm{~nm}$. Therefore, X-ray structural analyses may render a clue regarding the excimer formation. Notably, single crystal X-ray diffraction analysis, shown in Fig. 5 and S3, $\dagger$ indicated that two-dimensional intermolecular networks are formed by the face-to-edge stacking geometries in the crystal, and the step-like formation of $\mathbf{C z}^{\mathbf{9}} \mathbf{P h A n}$ was established unequivocally. Meanwhile, the peripheral carbazole moiety will also act as an intermolecular contact platform, adding further stabilization via the weak $\pi$ stacking interactions. ${ }^{22}$ These types of arrangement may be beneficial for the excimer formation in the solid state. ${ }^{23}$ As a comparison, the addition of a tert-butyl group on the anthracene moiety of $\mathbf{C z}^{\mathbf{9}} \mathbf{P h A n}$ forms $\mathbf{C z}^{\mathbf{9}} \mathbf{P h A n t}$ (see Scheme 1).

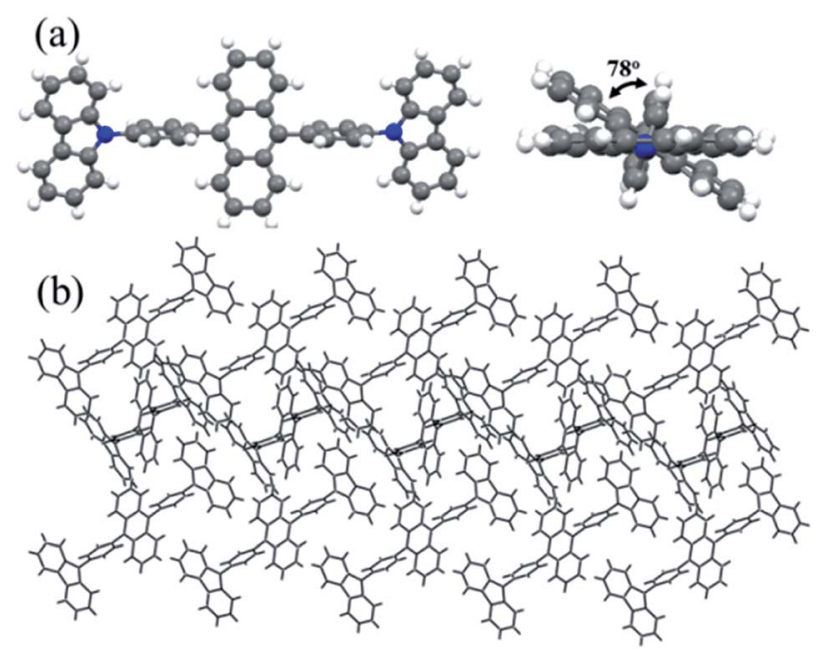

Fig. 5 X-ray crystallographic analysis of $\mathrm{Cz}^{9} \mathrm{PhAn}$. (a) Ball-and-stick models of $\mathrm{Cz}^{9} \mathrm{PhAn}$ viewed perpendicular to the anthracene plane (left) and along the carbazole plane (right). (b) Crystal packing diagram of $\mathrm{Cz}^{9} \mathrm{PhAn}$. 
$\mathbf{C z}^{9}$ PhAnt has been demonstrated as a blue emitter, ${ }^{20}$ exhibiting a normal emission (450 nm) only in solid film (see Fig. 1b). From the viewpoint of the chemical structure of $\mathbf{C z}^{\mathbf{9}} \mathbf{P h A n t}$, the steric effect of tert-butyl groups has proved to be an efficient methodology for reducing the intermolecular interaction and suppressing excimer emission. ${ }^{24}$ As a result, relatively loose molecular packing was resolved for $\mathbf{C z}^{\mathbf{9}} \mathbf{P h A n t}$, as indicated by the lack of excimer emission spectra. Obviously, removing the tert-butyl group at the 2-position of anthracene increases the strength of the intermolecular interaction between adjacent $\mathbf{C z}^{\mathbf{9}} \mathbf{P h A n}$ molecules, leading to good overlap of the vibronic wavefunction in the electronically excited state ${ }^{23 b}$ via the assistance of, e.g., lattice motion (vide supra).

The time-dependent DFT (B3LYP/6-31G(d,p)) calculations of $\mathbf{C z}^{9}$ PhAn showed the lowest lying transition to be the highest occupied molecular orbital (HOMO) $\rightarrow$ the lowest occupied molecular orbital (LUMO) in character, for which the HOMO and LUMO are mainly located at the anthracene moiety in both the monomer or the dimer-like structure truncated from the $\mathrm{X}$ ray crystal structure of $\mathbf{C z}^{\mathbf{9}} \mathbf{P h A n}$ (see Fig. S4†). Nevertheless, a small but non-negligible portion of the HOMO is located at the bridging phenyl ring. Careful examination of the X-ray structure of $\mathbf{C z}^{\mathbf{9}} \mathbf{P h A n}$ also indicates that the nearest anthracene (center) to anthracene (center) distance between two adjacent slabs is as far as $7.1 \AA$ (see Fig. 3b). Thus, the possibility of excimer formation originating from the anthracene*/anthracene $(*$ denotes the electronically excited state) pair is discarded. Alternatively, we noticed that the nearest distance between the phenyl bridge of one slab and center of anthracene of the adjacent slab is as short as $4.0 \AA$ (see Fig. 3b) while they are mutually twisted with a dihedral angle of $78^{\circ}$ (see Fig. 5). Additionally, the top view of the two slabs clearly shows that the center of anthracene is closely in line with the bridging phenyl plane (see Fig. S3†). The calculation shown in Fig. 3b also indicates the potential approach of frontier orbitals between the anthracine of one slab and the phenyl bridging ring of the adjacent slab. We thus tentatively propose an anthracene*/ phenyl ring excimer-like formation where the interaction is through an overlap between $\pi^{*}$ (anthracene) and $\pi$ (phenyl ring) orbitals in a face-to-edge-like orientation, the energy of which is then minimized by coupling the internal lattice motion that determines the rate of excimer formation. Note that in this case, the term "excimer" holds because the process still incorporates two $\mathbf{C z}^{\mathbf{9}} \mathbf{P h} \mathbf{A n}$ molecules in the excited and ground states, respectively. We realize that this type of face-to-edge excimer formation, though unusual, is plausible from the theoretical point of view. ${ }^{25}$

We then performed computation by utilizing the two truncated slabs of $\mathbf{C z}^{\mathbf{9}} \mathbf{P h A n}$ (see Fig. $3 \mathrm{~b}$ ) to gain further insight into the formation mechanism. Attempts by full geometry optimization (DFT at the B3LYP/6-31G(d,p) level and TD-DFT) in the excited state unfortunately failed, perhaps due to a rather small interaction between these two slabs. Alternatively, we then gradually decreased the parallel distance between two anthracene planes within the slabs, starting from $5.10 \AA$ (the X-ray data), by a decrement of $0.01 \AA$. According to the repulsion (attraction) of the potential energy surface in the ground (excited) states (see Fig. 3a), we may anticipate a smooth change or perhaps a (local) minimum of the $S_{0} \rightarrow S_{1}$ transition around the excimer formation region. As a result, at a parallel distance of $4.03 \AA$ between two slabs, a transition of $430 \mathrm{~nm} \mathrm{(23256}$ $\mathrm{cm}^{-1}$, see Fig. $\mathrm{S} 5 \dagger$ ) was calculated, for which the adjacent transition (with 0.01 A resolution) is higher in transition energy. This value is close to the onset of the excimer emission at $\sim 450$ $\mathrm{nm}$ (see the fitting curve in Fig. 1a). This approach, though being qualitative, supports the feasibility of excimer formation via certain lattice motions to shorten the inter-slab distance.

Experimentally, the HOMO levels were determined using ultraviolet photoelectron spectroscopy. The LUMO energy level was estimated from the HOMO level and the optical band gap. The corresponding HOMO/LUMO energy levels of $\mathbf{C z}^{\mathbf{9}} \mathbf{P h A n}$ were calculated to be $-5.83 /-2.95 \mathrm{eV}$. To further understand the charge-carrier transport properties, we used the time-offight (TOF) technique to evaluate the carrier mobilities. Representative TOF transients for holes of $\mathbf{C z}^{\mathbf{9}} \mathbf{P h A n}$ are revealed in Fig. 6a. Fig. 6b shows the hole mobility plotted as a function of the square root of the electric field. The hole mobility of $\mathrm{Cz}^{9} \mathbf{P h A n}$ lies in the range from $4.9 \times 10^{-4}$ to $4.5 \times 10^{-3} \mathrm{~cm}^{2} \mathrm{~V}^{-1}$ $\mathrm{s}^{-1}$ for fields varying from $4.5 \times 10^{5}$ to $7.9 \times 10^{5} \mathrm{~V} \mathrm{~cm}^{-1}$. In view of the dual emission (monomer and excimer) of $\mathbf{C} \mathbf{z}^{\mathbf{9}} \mathbf{P h A n}$, the application of this material as an emitter for a white OLED was investigated using a simple configuration: indium tin oxide (ITO)/HAT-CN (10 nm)/NPB (60 nm)/TCTA (5 nm)/Cz' PhAn (25 $\mathrm{nm}) / \mathrm{TPBI}(50 \mathrm{~nm}) / \mathrm{LiF}(0.5 \mathrm{~nm}) / \mathrm{Al}(100 \mathrm{~nm})$. To improve the hole injection from the anode, we used 4,5,8,9,11-hexaazatriphenylene-hexacarbonitrile (HAT-CN) ${ }^{\mathbf{2 6}}$ as a hole injection layer. Two hole-transporting layers (HTLs), which consisted of a $20 \mathrm{~nm}$-thick layer of 4,4'-bis[ $N$-(1-naphthyl)- $N$-phenyl]biphenyldiamine (NPB) ${ }^{27}$ and a $5 \mathrm{~nm}$-thick thick layer of $4,4^{\prime}, 4^{\prime \prime}$-tri $(N$ carbazolyl)triphenylamine (TCTA), ${ }^{28}$ were implemented. It is noteworthy that TCTA (HOMO level of $-5.7 \mathrm{eV}$ ) was inserted between NPB and $\mathbf{C z}^{\mathbf{9}} \mathbf{P h A n}$ with a stepwise increase in HOMOs into the emitting layer. To further confine the holes or the excitons generated within the emissive region, 1,3,5-tris $(N$ phenylbenzimidizol-2-yl)benzene (TPBI) ${ }^{29}$ with a high-energy gap was selected as the electron-transporting layer (ETL). LiF and $\mathrm{Al}$ served as an electron-injecting layer and cathode, respectively.

Devices employing $\mathbf{C z} \mathbf{z}^{\mathbf{P}} \mathbf{P h A n}$ as an emitter are compared in Fig. 7. The $\mathbf{C z}^{\mathbf{9}} \mathbf{P h A n}$ device displayed a turn-on voltage of $2.8 \mathrm{~V}$,
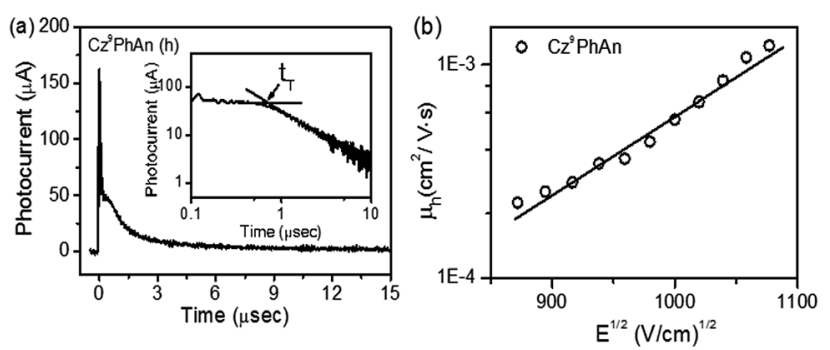

Fig. 6 (a) Representative TOF transients for $\mathrm{Cz}^{9} \mathrm{PhAn}$ (thickness: 1.55 $\mu \mathrm{m} ; E=4.5 \times 10^{5} \mathrm{~V} \mathrm{~cm}^{-1}$ ). Inset: double logarithmic plot. (b) Hole mobility of $\mathrm{Cz}^{9} \mathrm{PhAn}$ plotted with respect to $E^{1 / 2}$. 

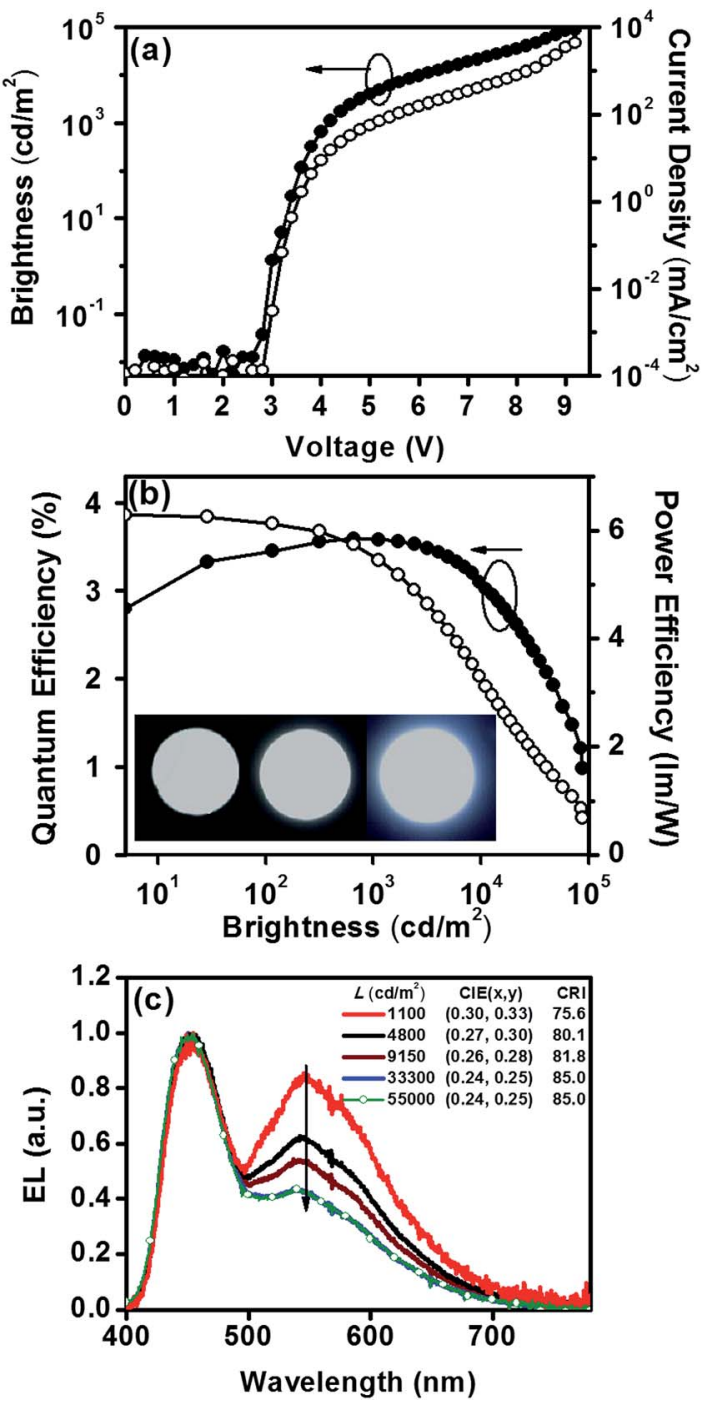

Fig. 7 (a) Current density-voltage-luminance $(J-V-L)$ characteristics, (b) external quantum ( $\left.\eta_{\text {ext }}\right)$ and power efficiencies $\left(\eta_{\mathrm{P}}\right)$ as a function of brightness, and (c) normalized EL spectra of the white device.

and the driving voltage of the WOLED at $1000 \mathrm{~cd} \mathrm{~m}^{-2}$ was about $4.2 \mathrm{~V}\left(15 \mathrm{~mA} \mathrm{~cm}^{-2}\right)$, achieving a maximum external quantum efficiency $\left(\eta_{\text {ext }}\right)$ of $3.6 \%$, corresponding to a current efficiency $\left(\eta_{\mathrm{c}}\right)$ of $8.5 \mathrm{~cd} \mathrm{~A}^{-1}$ and power efficiency $\left(\eta_{\mathrm{p}}\right)$ of $6.3 \mathrm{~lm} \mathrm{~W}^{-1}$. The EL emission spectra had two distinct emission bands, a blue band (monomer) centered at $453 \mathrm{~nm}$ and a broad yellow band (excimer) with a peak at ca. $545 \mathrm{~nm}$, and demonstrated white emission with a high color-rendering index (CRI = 75.6-85.0), as shown in Fig. 7c. The intensity ratio for the excimer versus normal emission decreased with increasing brightness from 1100 to $33300 \mathrm{~cd} \mathrm{~m}^{-2}$, and then remained unchanged up to $55000 \mathrm{~cd} \mathrm{~m}^{-2}$ (see Fig. 7c) and even higher, leading to a slight shift of the CIE coordinates from $(0.30,0.33)$ to $(0.24,0.25)$. The driving-voltage-dependent emission ratio is commonly interpreted by mismatch between the electron and hole transfer rates in the active layer, resulting in an uneven exciton distribution. However, this normally occurs in the host-dopant system, ${ }^{8}$ which may not be operative for the non-dopant system in this study. Alternatively, because the excimer is in the lower energy level, the cascade from the blue (monomer) emitter to the yellow (excimer) might account for the ratiometric change of dual emission. Under lower current (low brightness), the emission ratio is driving-voltage-dependent, while it remains unchanged upon reaching saturation at high current (high brightness). Remarkably, the device showed rather low efficiency roll-off at a brightness of $1000 \mathrm{~cd} \mathrm{~m}^{-2}$, while the recorded $\eta_{\text {ext }}$ remained as high as $3.6 \%$ with CIE coordinates of $(0.30$, 0.33). Angle-dependent EL intensity measurements show the EL intensity distributions of the device as well as the ideal Lambertian distribution for comparison (Fig. 8).

Considering the PLQY of 0.47 , a singlet ratio around $25 \%$, and a usual optical out-coupling efficiency of $20 \%$ for typical OLEDs, one would only expect an upper limit of $c a$. $2.4 \%$ in $\eta_{\text {ext }}$ with such a PLQY. Careful analysis indicates that this superior efficiency is attributed to the preferred horizontal orientation of the transition (emitting) dipoles that would significantly enhance the light outcoupling efficiency $\left(\eta_{\text {out }}\right)$ of the OLED. ${ }^{30}$ Fig. 9 shows the anisotropies in the refractive index and extinction coefficient as a function of wavelength. The extinction coefficient of the out-of-plane (vertical) component (blue line) is small compared to that of the in-plane (horizontal) component (black line). This result indicates that the transition dipole moments of $\mathbf{C z}^{\mathbf{9}} \mathbf{P h A n}$ are mainly oriented horizontally, leading to a high optical out-coupling effect when emitting light from such dipoles. According to previous studies, ${ }^{30}$ highly horizontally oriented emitting dipoles could result in an up to 2fold out-coupling enhancement, compared to isotropic emitters.

To gain further insight, the Grazing Incidence Wide Angle Xray scattering (GIWAXS) pattern of the $\mathbf{C z}^{\mathbf{9}} \mathbf{P h A n}$ thin film is shown in Fig. 10. A broad diffraction ring is seen for the film, as indicated by the red arrow. The corresponding $d$-spacing is $4.0 \AA$ for $\mathbf{C z}^{\mathbf{9}} \mathbf{P h A n}$, which can be rationally assigned to the periodical face-to-edge stacking of the anthracene and phenyl ring. Furthermore, the film shows an uneven diffraction ring with stronger intensity at the $q_{z}$ axis, implying a preferential stacking of the molecules along the thickness direction of the film, which evidences the horizontal orientation of the transition dipoles that enhances the light outcoupling efficiency.

The facile synthetic scheme with high product yield $(>70 \%)$ makes it feasible to scale-up the synthesis of $\mathbf{C z}^{\mathbf{9}} \mathbf{P h A n}$ to hundreds of grams in the lab. Using this approach, we then

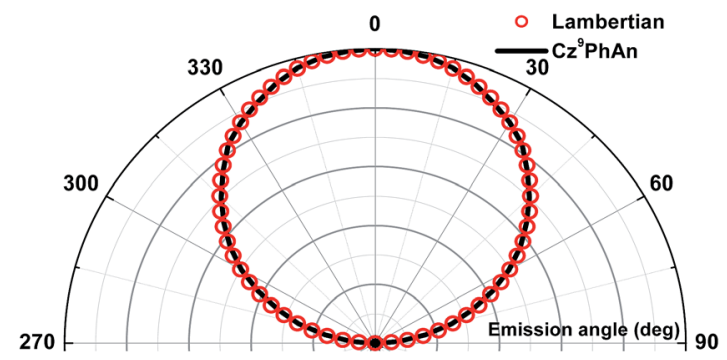

Fig. 8 Angular distribution of radiation irradiance for the white device and the Lambertian emission pattern (normalized to the $0^{\circ}$ irradiance). 


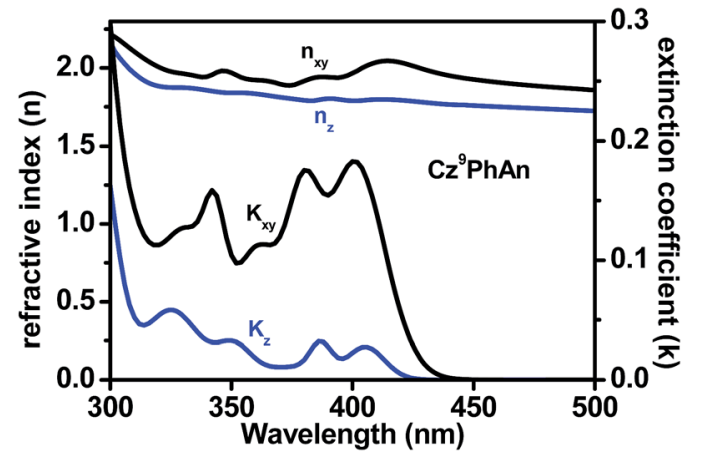

Fig. 9 Optical anisotropy of the $\mathrm{Cz}^{9} \mathrm{PhAn}$ film: anisotropic refractive indices $n$ and extinction coefficients $k$. The black lines $\left(n_{x y}\right.$ and $\left.k_{x y}\right)$ and blue lines $\left(n_{z}\right.$ and $\left.k_{z}\right)$ indicate the in-plane (horizontal) and out-ofplane (vertical) components of the optical constants, respectively.

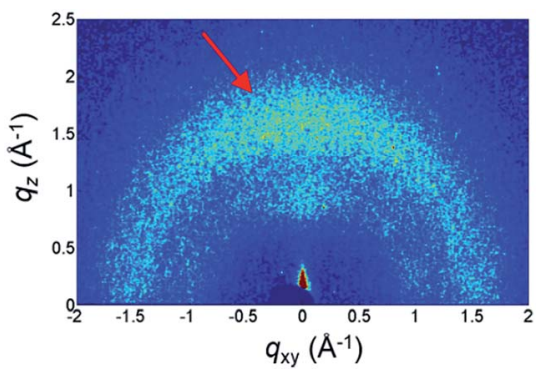

Fig. 10 GIWAXS pattern of $\mathrm{Cz}^{9} \mathrm{PhAn}$ thin film. The arrow indicates the diffraction ring of the face-to-edge stacking.

further fabricated several prototype lighting devices with active illuminating areas of either $5 \times 10 \mathrm{~cm}^{2}$ or $5 \times 5 \mathrm{~cm}^{2}$. The resulting WOLEDs, shown in Fig. S6 of the ESI, $\dagger$ demonstrate the great potential of this single-molecule-active-layered, panchromatic WOLED for pragmatic application.

\section{Conclusions}

To sum up, a new single white light-emitting material, $\mathbf{C z}^{\mathbf{9}} \mathbf{P h A n}$, was designed and synthesized. The tight intermolecular packing, resulting from the face-to-edge interactions between the phenyl bridge of one slab and the center of anthracene of the adjacent slab, is capable of producing excimer emission. Consequently, the panchromatic emission of $\mathbf{C z}^{\mathbf{9}} \mathbf{P h A n}$ is contributed by its normal (blue) and excimer (yellow) fluorescence, as supported by the combination of X-ray analyses and the corresponding photophysical studies. A single-component WOLED incorporated with $\mathbf{C z}^{\mathbf{9}} \mathbf{P h A n}$ as an emitter exhibits a high value of $\eta_{\text {ext }}$ of $3.6 \%$ at $1000 \mathrm{~cd} \mathrm{~m}^{-2}(4.2 \mathrm{~V}$ ) with CIE coordinates of $(0.30,0.33)$. The $\mathbf{C z}^{\mathbf{9}} \mathbf{P h A n}$ film revealed a preferred orientation of the transition dipole moment in the emitting layer to enhance light outcoupling. Knowing that the excimer formation commonly originates from $\pi$-stacking of the framework in the film, the enhanced light outcoupling in the device is expected, which is exactly what we observed in this study. The non-doped $\mathbf{C z}^{\mathbf{9}} \mathbf{P h A n}$ WOLED demonstrates that fluorescent white light generation can be achieved in a singlemolecule system with pragmatic WOLED performance, which is certainly competitive with other contemporary display technologies in terms of simplicity and hence cost-effectiveness and reliability. Our comprehensive structural and spectroscopic/ dynamics study of the excimer emission and the record high efficiency of the single-molecule type fluorescent WOLED are to be far-reaching in the future due to the in-depth understanding of mechanism acquired in this study.

\section{Acknowledgements}

The authors thank the Ministry of Science and Technology, Taiwan, for funding support.

\section{References}

1 (a) G. Zhou, W. Y. Wong and S. Suo, J. Photochem. Photobiol., C, 2010, 11, 133; (b) Q. Wang and D. Ma, Chem. Soc. Rev., 2010, 39, 2387; (c) G. M. Farinola and R. Ragni, Chem. Soc. Rev., 2011, 40, 3467.

2 Y. R. Sun, N. C. Giebink, H. Kanno, B. W. Ma, M. E. Thompson and S. R. Forrest, Nature, 2006, 440, 908.

3 G. Zhou, Q. Wang, C.-L. Ho, W.-Y. Wong, D. Ma and L. Wang, Chem. Commun., 2009, 3574.

4 S.-H. Cheng, W.-Y. Hung, M.-H. Cheng, H.-F. Chen, G.-H. Lee, C.-L. Chung, T.-C. Yeh, W.-C. Tang, S.-L. Huang and K.-T. Wong, Adv. Electron. Mater., 2015, 1500241.

5 S. H. Kim, S. Park, J. E. Kwon and S. Y. Park, Adv. Funct. Mater., 2011, 21, 644.

6 S. Park, J. E. Kwon, S. H. Kim, J. Seo, K. Chung, S. Y. Park, D. J. Jang, B. M. Medina, J. Gierschner and S. Y. Park, J. Am. Chem. Soc., 2009, 131, 14043.

7 H.-C. Peng, C.-C. Kang, M.-R. Liang, C.-Y. Chen, A. Demchenko, C.-T. Chen and P.-T. Chou, ACS Appl. Mater. Interfaces, 2011, 3, 1713.

8 (a) X. Yang, Z. Wang, S. Madakuni, J. Li and G. E. Jabbour, Adv. Mater., 2008, 20, 2405; (b) T. Fleetham, J. Ecton, Z. Wang, N. Bakken and J. Li, Adv. Mater., 2013, 25, 2573.

9 (a) Y. I. Park, O. Postupna, A. Zhugayevych, H. Shin, Y.-S. Park, B. Kim, H.-J. Yen, P. Cheruku, J. S. Martinez, J. W. Park, S. Tretiak and H.-L. Wang, Chem. Sci., 2015, 6, 789; (b) A. Ozawa, A. Shimizu, R. Nishiyabu and Y. Kubo, Chem. Commun., 2015, 51, 118; (c) X.-H. Jin, C. Chen, C.-X. Ren, L.-X. Cai and J. Zhang, Chem. Commun., 2014, 50, 15878.

10 K. C. Tang, M. J. Chang, T. Y. Lin, H. A. Pan, T. C. Fang, K. Y. Chen, W. Y. Hung, Y. H. Hsu and P. T. Chou, J. Am. Chem. Soc., 2011, 133, 17738.

11 J. Y. Li, D. Liu, C. Ma, O. Lengyel, C. S. Lee, C. H. Tong and S. T. Lee, Adv. Mater., 2004, 16, 1538.

12 Y. Liu, M. Nishiura, Y. Wang and Z. M. Hou, J. Am. Chem. Soc., 2006, 128, 5592.

13 R. M. Adhikari, L. Duan, L. Hou, Y. Qiu, D. C. Neckers and B. K. Shah, Chem. Mater., 2009, 21, 4638.

14 L. Wang, M. F. Lin, W. K. Wong, K. W. Cheah, H. L. Tam, Z. Q. Gao and C. H. Chen, Appl. Phys. Lett., 2007, 91, 183504. 
15 S. Tao, Y. Zhou, C. S. Lee, S. T. Lee, D. Huang and X. Zhang, J. Mater. Chem., 2008, 18, 3981.

16 L. Wang, W. Y. Wong, M. F. Lin, W. K. Wong, K. W. Cheah, H. L. Tam and C. H. Chen, J. Mater. Chem., 2008, 18, 4529.

17 K. Danel, T. H. Huang, J. T. Lin, Y. T. Tao and C. H. Chuen, Chem. Mater., 2002, 14, 3860.

18 Y. H. Kim, H. C. Jeong, S. H. Kim, K. Yang and S. K. Kwon, Adv. Funct. Mater., 2005, 15, 1799.

19 Y. J. Pu, A. Kamiya, K. I. Nakayama and J. Kido, Org. Electron., 2010, 11, 479.

20 Y. H. Chen, S. L. Lin, Y. C. Chang, Y. C. Chen, J. T. Lin, R. H. Lee, W. J. Kuo and R. J. Jeng, Org. Electron., 2012, 13, 43.

21 Y. C. Chang, S. C. Yeh, Y. H. Chen, C. T. Chen, R. H. Lee and R. J. Jeng, Dyes Pigm., 2013, 99, 577.

22 J. Hu, Y. Pu, Y. Yamashita, F. Satoh, S. Kawata, H. Katagiri, H. Sasabe and J. Kido, J. Mater. Chem. C, 2013, 1, 3871.

23 (a) G. Zhang, G. Yang, S. Wang, Q. Chen and J. S. Ma, Chem.Eur. J., 2007, 13, 3630; (b) T. Hinoue, Y. Shigenoi, M. Sugino, Y. Mizobe, I. Hisaki, M. Miyata and N. Tohnai, Chem.-Eur. J., 2012, 18, 4634; (c) P. C. Nandajan, P. P. Neelakandan and D. Ramaiah, RSC Adv., 2013, 3, 5624.
24 (a) L. S. Hung and C. H. Chen, Mater. Sci. Eng., R, 2002, 39, 143; (b) A. C. Benniston, A. Harriman, S. L. Howell, C. A. Sams and Y. G. Zhi, Chem.-Eur. J., 2007, 13, 4665.

25 L. A. Dissado and S. H. Walmsley, Chem. Phys., 1984, 86, 375.

26 Y. K. Kim, J. W. Kim and Y. Park, Appl. Phys. Lett., 2009, 94, 063305.

27 D. H. Lee, Y. P. Liu, K. H. Lee, H. Chae and S. M. Cho, Org. Electron., 2010, 11, 427.

28 (a) S. J. Su, E. Gonmori, H. Sasabe and J. Kido, Adv. Mater., 2008, 20, 4189; (b) Y. Y. Lyu, J. Kwak, W. S. Jeon, Y. Byun, H. S. Lee, D. Kim, C. Lee and K. Char, Adv. Funct. Mater., 2009, 19, 420.

29 S. J. Yeh, M. F. Wu, C. T. Chen, Y. H. Song, Y. Chi, M. H. Ho, S. F. Hsu and C. H. Chen, Adv. Mater., 2005, 17, 285.

30 (a) S. Y. Kim, W. I. Jeong, C. Mayr, Y. S. Park, K. H. Kim, J. H. Lee, C. K. Moon, W. Brütting and J. J. Kim, Adv. Funct. Mater., 2013, 23, 3896; (b) Y. S. Park, S. Lee, K. H. Kim, S. Y. Kim and J. J. Kim, Adv. Funct. Mater., 2013, 23, 4914; (c) S. Lee, K. H. Kim, D. Limbach, Y. S. Park and J. J. Kim, Adv. Funct. Mater., 2013, 23, 4105; (d) S. Lee, D. Limbach, K. H. Kim, S. J. Yoo, Y. S. Park and J. J. Kim, Org. Electron., 2013, 14, 1856. 